\title{
DESIGN OF THE SUPERCONDUCTING SECTION OF THE SPL LINAC AT CERN
}

\author{
F. Gerigk, M. Vretenar, CERN, Geneva, Switzerland \\ R.D. Ryne, LBNL, Berkeley, USA
}

\begin{abstract}
In order to set up a powerful proton source for a future Neutrino Factory, increasing at the same time the flux of protons available for new and existing facilities, CERN is studying a $2.2 \mathrm{GeV}$ superconducting $\mathrm{H}^{-}$linac for $4 \mathrm{MW}$ beam power, called SPL. The superconducting part of this linac covers the energy range from $120 \mathrm{MeV}$ to $2.2 \mathrm{GeV}$. Three sections made of $352 \mathrm{MHz}$ cavities with nominal $\beta$ of $0.52,0.7$ and 0.8 bring the beam energy up to $1 \mathrm{GeV}$. From this energy, superconducting cavities from LEP, or $\beta=0.8$ cavities, can be used to reach the final energy of $2.2 \mathrm{GeV}$. This paper covers the optimisation for the superconducting part, the beam dynamics design principles, the matching between sections, and the results of multiparticle simulations with up to 50 million particles. To demonstrate the stability of the design, matched and mismatched input beams are used.
\end{abstract}

\section{INTRODUCTION}

The SPL is a high intensity $\mathrm{H}^{-}$linac designed for an energy of $2.2 \mathrm{GeV}$ and a beam power of $4 \mathrm{MW}$ [1]. It is proposed as a new injector for the CERN proton complex, with the aim to improve the beams delivered to the CERN users, and to be the proton driver for a future radioactive beam facility and for a high-intensity Neutrino Factory [2]. The high energy part of this linac makes use of the superconducting cavities recuperated from the decommissioned LEP collider. The RF frequency for the linac has been fixed at $352 \mathrm{MHz}$, in order to use the LEP klystrons and other RF components. Table 1 summarises the main linac design parameters.

Table 1: Main linac design parameters

\begin{tabular}{|c|c|c|}
\hline Particles & $\mathrm{H}^{-}$ & \\
\hline Output energy & 2.2 & $\mathrm{GeV}$ \\
\hline Mean current during pulse & 13 & $\mathrm{~mA}$ \\
\hline Max. bunch current & 22 & $\mathrm{~mA}$ \\
\hline Duty cycle & 14 & $\%$ \\
\hline Mean beam power & $\overline{4}$ & $\overline{M W}$ \\
\hline Repetition frequency & 50 & $\mathrm{~Hz}$ \\
\hline Beam pulse duration & 2.8 & $\mathrm{~ms}$ \\
\hline No. of particles per pulse & $2.27 \times 10^{14}$ & $\mathrm{H}^{-} /$pulse \\
\hline RF frequency & 352.2 & $\mathrm{MHz}$ \\
\hline$\varepsilon_{\text {transv }, \text { r.m.s.,norm. }}$ & 0.4 & $\pi \mathrm{mm} \mathrm{mrad}$ \\
\hline$\varepsilon_{\text {long.,r.m.s. }, \text { norm }}$ & 0.3 & $\pi^{o} \mathrm{MeV}$ \\
\hline & 0.755 & $\pi \mathrm{mm} \mathrm{mrad}$ \\
\hline
\end{tabular}

\section{LAYOUT OF THE SUPERCONDUCTING SECTION}

Starting at $120 \mathrm{MeV}$, three or four families of superconducting cavities are foreseen. A first section of 4-cell $\beta=0.52$ cavities made of bulk niobium brings the beam to $236 \mathrm{MeV}$, followed by a section of 4-cell cavities with a nominal $\beta$ of 0.7 , which is the minimum $\beta$ for which the standard CERN technique of sputtering niobium on copper can be applied [3]. At $380 \mathrm{MeV}$ a section of 5-cell $\beta=0.8$ cavities starts. Their cryostats [4] and cut-off tubes with all the ancillary equipment (input and HOM couplers) can be recuperated from LEP. Above $1.1 \mathrm{GeV}$ two options have then been considered to reach the final energy [5], either to pass to LEP cavities, or to continue with the $\beta=0.8$ cavities, more efficient in terms of gradient and transit time factor but more expensive to produce. These two options are indicated as (a) and (b) in Table 2, which summarises the main design parameters for the superconducting section.

Table 2: Main layout parameters

\begin{tabular}{|c|c|c|c|c|c|}
\hline $\boldsymbol{\beta}$ & $\begin{array}{c}\mathbf{W}_{\text {out }} \\
{[\mathbf{M e V}]}\end{array}$ & $\begin{array}{c}\mathbf{E}_{\mathbf{0}} \mathbf{T} \\
{[\mathbf{M V} / \mathbf{m}]}\end{array}$ & $\begin{array}{c}\text { No. of } \\
\text { cavities }\end{array}$ & $\begin{array}{c}\text { No. of RF } \\
\text { sources }\end{array}$ & $\begin{array}{c}\text { length } \\
{[\mathbf{m}]}\end{array}$ \\
\hline 0.52 & 236 & 3.5 & 42 & 42 tetrodes & 101 \\
\hline 0.7 & 383 & 5 & 32 & 32 tetrodes & 80 \\
\hline 0.8 & 1111 & 9 & 52 & 13 klystrons & 166 \\
\hline \hline 1.0 (a) & 2204 & 7.5 & 104 & 18 klystrons & 324 \\
\hline \hline 0.8 (b) & 2235 & 9 & 76 & 19 klystrons & 237 \\
\hline
\end{tabular}

To reduce the effect of cavity vibrations on the beam, the more sensitive low- $\beta$ cavities are fed by individual tetrode amplifiers, while in the high-energy sections four $\beta=0.8$ or six $\beta=1$ cavities are fed by one LEP klystron.

The use of modern surface processing techniques allows a higher gradient for the $\beta=0.8$ cavities: $9 \mathrm{MV} / \mathrm{m}$ instead of the $7.5 \mathrm{MV} / \mathrm{m}$ achieved in LEP operation. Together with the considerably higher transit time factor of the $\beta=0.8$ cavities between 1.1 and $2.2 \mathrm{GeV}$, version (b) becomes $87 \mathrm{~m}$ shorter than version (a). Due to the higher peak power per cavity the number of cavities per klystron can be reduced from six in version (a) to four in version (b), simplifying the vector sum compensation of cavity errors. Furthermore a new cavity design permits moving the mechanical resonances away from the linac repetition frequency. Since the estimated difference in cost between the two options is negligible ( $2 \%$ of the overall linac cost) [5], the $\beta=0.8$ version has now been assumed as the reference layout, and the following considerations will refer to it. 


\section{BEAM DYNAMICS}

Quadrupole doublets with an aperture of $120 \mathrm{~mm}$ provide the transverse focusing throughout the linac. The maximum number of $\beta \lambda$ per focusing period varies between 17 in the $\beta=0.52$ section ( $\Rightarrow$ three 4 -cell cavities) and 33 in the $\beta=0.8$ section. Due to the low longitudinal phase advance in the high energy part, the length of the focusing periods was doubled from $16.5 \beta \lambda$ below $1.1 \mathrm{GeV}$ to $33 \beta \lambda$ above $1.1 \mathrm{GeV}$, thus providing space for two cryostats with altogether eight 5-cell cavities.

All multiparticle simulations were carried out at $40 \mathrm{~mA}$, twice the nominal current, using the parallel 3D PIC code IMPACT [6]. For matching and designing the linac a modified version of the r.m.s. envelope code FIX3D [7] was employed. Since IMPACT offers the capability to simulate a beam with up to $10^{8}$ particles $(25 \%$ of the actual number of particles in an SPL bunch!), several runs were made to determine the number of particles which is actually necessary for meaningful results. Figure 1 shows the results for runs with a mismatched input beam (30\% quadrupolar mismatch. The variation in transverse emittance with respect to a run with 50 million particles is plotted. While the im-

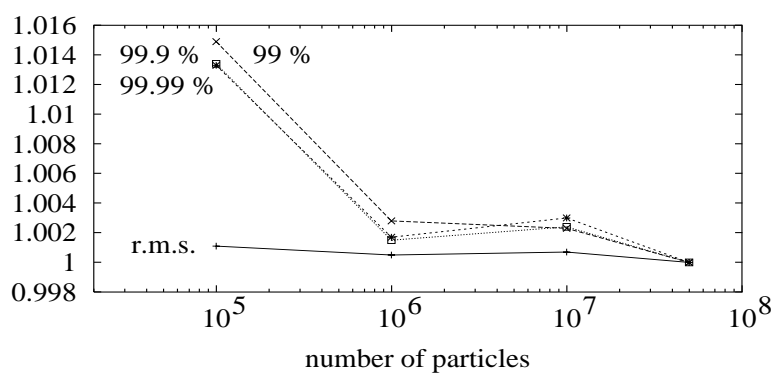

Figure 1: Relative variation of transverse emittance as a function of the number of particles in a computation with $30 \%$ quadrupolar mismatch

provement from $10^{5}$ to $10^{6}$ particles is clearly visible, an even higher number of particles does not seem to improve the accuracy of the results. A similar study was made to determine the size of an appropriate space charge grid. Runs with $10^{6}$ particles and grids between $32^{3}$ and $256^{3}$ showed less than $0.7 \%$ variation of the $99.99 \%$ emittance between the $128^{3}$ and the $256^{3}$ grid. Finally we fixed the simulation parameters to $10^{6}$ particles on a $128^{3}$ space charge grid.

\subsection{MATCHING AND PHASE SLIP}

Multi cell cavities which are built for a given particle velocity and operated over a range of velocities, provide a single cell "synchronous phase" for the beam that deviates from the "average phase" of the cavity (Fig.2). This phase slippage reduces the transit time factors, the longitudinal focusing forces, and the energy acceptance of the machine. The reduction of the energy acceptance (the height of the $\mathrm{RF}$ bucket) is determined by the reduced transit time factor times an additional "slip factor", which accounts for the nonlinear dependence of the focusing forces on the slip angle [8]. When simulating multi cell structures with large slip angles ( $\pm 55^{\circ}$ in our case), the "slip factor" is often ignored, which yields wrong phase advance values, incorrect magnet settings and cavity phasing, and thus increased mismatch at every transition between sections. Therefore, special care was taken to ensure that the matching code and the multiparticle code used the same RF gap model and the same method of dealing with phase slippage. In our case both codes make use of the on-axis field maps as calculated by SUPERFISH [9] and apply the correct single cell phases that are seen by the beam (see Fig. 2).

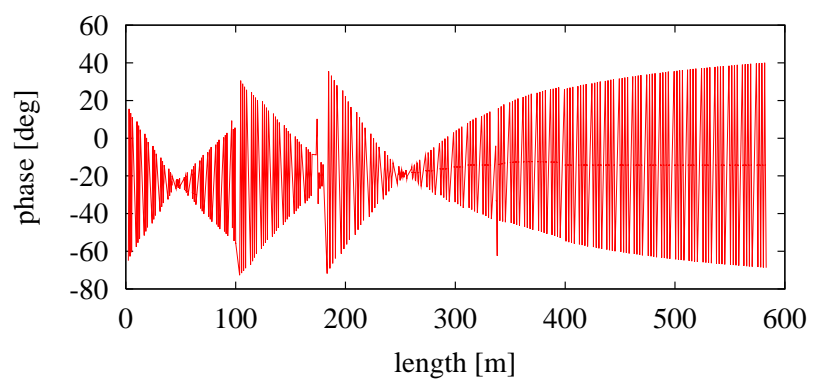

Figure 2: Mean bunch phase at cell centres along the linac

The matching between sections is achieved by varying existing beam line elements before and after the transition.

\subsection{DESIGN PRINCIPLES}

The design is based on a systematic evaluation of different settings for the matched phase advance values of each focusing period. By testing several optics for their stability to a mismatched input beam, we found that the tune ratios and their location in the "Hofmann Charts" [10] provide an important guideline for the beam dynamics layout. In the final design we avoid the unstable regions of the chart by adjusting the tune ratios appropriately. The transitions between sections are designed to keep the phase advance per meter as smooth as possible (Fig.3). Due to the changing length of the focusing periods at transitions this results in
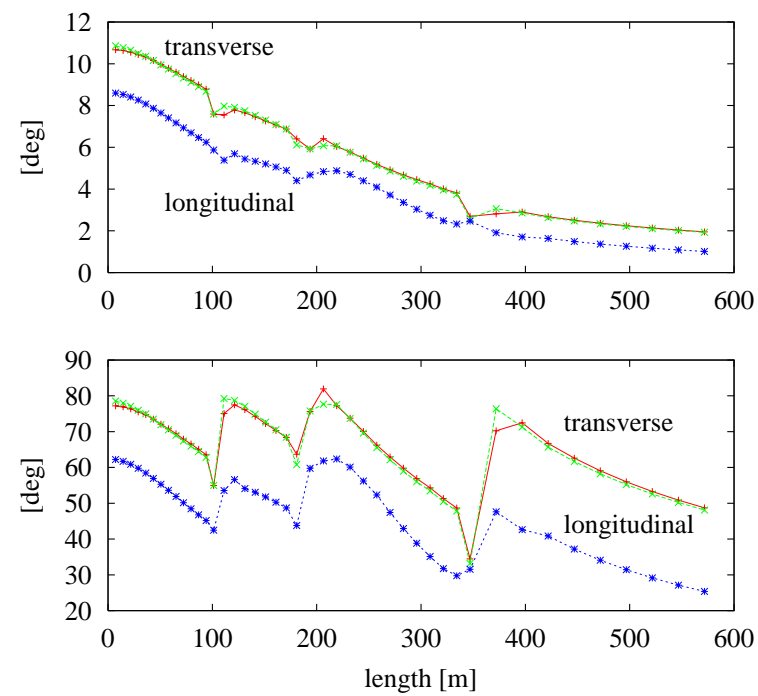

Figure 3: Zero current phase advance per meter (upper) and per period (lower) 
substantial but innocuous jumps of the phase advance per period. Despite these jumps, the zero current phase advance is always kept below $90^{\circ}$ (Fig.3). The ratio between longitudinal and transverse "beam temperature" varies between 1.6 in the beginning and 0.8 towards the end of the linac.

\subsection{SIMULATION RESULTS}

In the matched case there is practically no emittance growth, even the $99.99 \%$ emittance stays fairly constant (Fig. 4). The ratio between the minimum beam pipe ra-
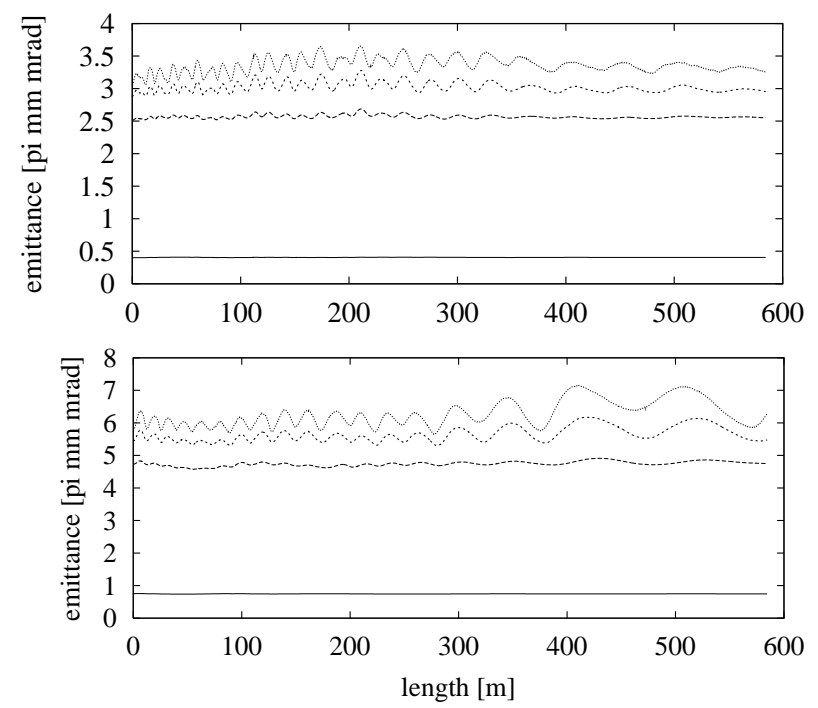

Figure 4: Transverse (upper) and longitudinal (lower) emittances for the matched case in ascending order: r.m.s., 99\%, 99.9\%, and 99.99\%

dius and the r.m.s. beam size varies between 16 and 24 , providing enough safety margin against machine activation by lost particles.

The stability of the design was tested with mismatched input beams, following the approach given in [11]. For bunched beams, every mismatch can be decomposed into three eigenmodes: the Quadrupolar mode, the Highfrequency, and the Low-frequency mode. By changing the input Twiss parameters $\alpha$ and $\beta$ by the same amount one can excite the three modes separately with a maximum amplitude at the beginning of the linac. This excitation is independent of the position where the mismatch is induced and provides a reasonable criterion for comparing mismatched beams in different designs. The three eigenmodes are excited with the largest oscillation showing a $30 \%$ mismatch.

In case of the Quadrupolar and the High-frequency mode the dominant mismatch amplitudes are found in the transverse planes, the planes where the highest emittance growth rates are observed (Table 3 ). In spite of the strong mismatch, the transverse r.m.s. emittance growth never exceeds $20 \%$. The highest longitudinal emittance growth $(7 \%$ r.m.s.) is found for Low-frequency mode excitation, where the dominant mismatch oscillation occurs in the longitudinal plane. The $99.99 \%$ emittances in Table 3 indicate the formation of beam halo for strong mismatch. However, even in the worst case (High-frequency mode excitation) the $100 \%$ transverse beam radius remains below $16 \mathrm{~mm}$, providing sufficient safety margin with respect to the minimum beam pipe radius at the quadrupoles of $60 \mathrm{~mm}$.

Table 3: Maximum emittance growth rates for matched and mismatched input beams

\begin{tabular}{|l|c|c|c|c|}
\hline & matched & Quad. & High & Low \\
\hline$\varepsilon_{r . m . s ., x / y}$ & $1.03 / 1.01$ & $1.18 / 1.19$ & $1.08 / 1.04$ & $1.04 / 1.02$ \\
$\varepsilon_{r . m . s ., z}$ & 1.00 & 1.00 & 1.01 & 1.07 \\
$\varepsilon_{99 \%, x / y}$ & $1.06 / 1.07$ & $2.19 / 2.48$ & $1.23 / 1.14$ & $1.06 / 1.05$ \\
$\varepsilon_{99 \%, z}$ & 1.04 & 1.04 & 1.13 & 1.75 \\
$\varepsilon_{99.99 \%, x / y}$ & $1.21 / 1.20$ & $4.68 / 5.20$ & $9.90 / 2.15$ & $1.21 / 1.23$ \\
$\varepsilon_{99.99 \%, z}$ & 1.24 & 1.21 & 1.41 & 5.14 \\
\hline
\end{tabular}

\section{CONCLUSIONS}

The code IMPACT with 1 million particles and an appropriate space charge grid of $128^{3}$ provides a powerful tool for studying the stability of the beam dynamics design and the possible halo formation due to mismatched input beams. In the case of the SPL superconducting section, a careful design that follows some basic rules and that avoids space charge resonances by a proper selection of the working point in the "Stability Charts" developed by Hofmann [10], shows minimum emittance growth in the presence of mismatch. The energy spread in the presence of mismatch is appropriate for loss free ring injection.

\section{ACKNOWLEDGEMENTS}

This research used resources of the National Energy Research Scientific Computing Center.

\section{REFERENCES}

[1] Ed. M. Vretenar. Conceptual Design of the SPL, a HighPower Superconducting $\mathrm{H}^{-}$Linac at CERN,CERN 2000012.

[2] R. Garoby. Current Activities for a Neutrino Factory at CERN, CERN/PS-RF 2001-007. In Proc. HEACC, 2001.

[3] R. Losito. CERN SL-Note-2000-047-CT. Geneva.

[4] C. Benvenuti et al. Production and Test of $352 \mathrm{MHz}$ Niobium-Sputtered Reduced- $\beta$ Cavities. In Proc. 8'th Worksh. on RF Superconductivity, Abano Terme, Italy, page 1038, 1997.

[5] F. Gerigk. CERN-OPEN-2001-024. Geneva.

[6] J. Qiang; R.D. Ryne; S. Habib; V. Decyk. An ObjectOriented Parallel Particle-In-Cell Code for Beam Dynamics Simulation in Linear Accelerators. Journal of Computational Physics, 163(2):434-451, 9/2000.

[7] R.D. Ryne. LA-UR-95-391. LANL, Los Alamos, 1995.

[8] F. Gerigk. CERN-NUFACT-NOTE 2001-072. Geneva.

[9] J.H. Billen; L.M. Young. LA-UR-96-1834. LANL.

[10] F. Gerigk; I. Hofmann. this conference.

[11] A. Letchford; K. Bongardt; M. Pabst. Halo Formation of Bunched Beams in Periodic Focusing Systems. In Proc. of PAC 1999, page 1767. PAC, 1999. 